\title{
CARDIOEMBOLIC STROKE: RISK FACTORS, CliniCAL FEATURES, AND EARLY OUTCOME IN 956 CONSECUTIVE PATIENTS
}

\author{
Kilian Griñán ${ }^{1}$, Adrià Arboix ${ }^{1 *}$, Joan Massons ${ }^{1}$, LAURa DíEZ ${ }^{1}$, EnRic Vergés ${ }^{1}$, Francisco GiL ${ }^{1}$, \\ Jordi ArbolX-Alió ${ }^{2}$, MARÍA-José SÁNCHEZ-LÓPEZ ${ }^{3}$, AND LuÍS GARCíA-EROLES ${ }^{4}$ \\ ${ }^{1}$ Cerebrovascular Division, Department of Neurology, Hospital Universitari Sagrat Cor, Barcelona; ${ }^{2}$ Department \\ of Sports Sciences, Ramon Llull University, Faculty of Psychology, Education and Sport Sciences (FPCEE), Blanquerna, \\ Barcelona; ${ }^{3}$ Library, Hospital Universitari Sagrat Cor, Universitat de Barcelona, Barcelona; ${ }^{4}$ Organization and \\ Information Systems, Northern Metropolitan Territorial Management, Institut Català de la Salut, Barcelona, Spain
}

\begin{abstract}
Background: There is little information about the early clinical features of cardioembolic stroke before complementary examinations. Objective: The aim of this study was to identify risk factors, clinical features, and early outcomes of cardioembolic stroke. Methods: Retrospective study based on prospectively collected data available from a university medical center hospitalbased stroke registry. Consecutive patients diagnosed with cardioembolic infarction were selected and compared to those diagnosed with an atherothrombotic stroke. Predictors of cardioembolic infarction were assessed by multivariate analysis. Results: From a cohort of 4597 consecutive patients, we studied 956 patients diagnosed with cardioembolic infarction (80 years [standard deviation (SD) 9.14]; 63\% women) and 945 with atherothrombotic infarction (77.01 years [SD 9.75]; 49.8\% women). The univariate comparative analysis reported that advanced age ( $\geq 85$ years), female gender, atrial fibrillation (AF), ischemic heart disease, and congestive heart failure were significantly more frequent in the cardioembolic group, whereas hypertension, diabetes, peripheral vascular disease, heavy smoking, hyperlipidemia, and previous transient ischemic attack were significant in the atherothrombotic group. In the logistic regression model, AF (odds ratio [OR] 15.75, 95\% confidence interval [Cl]: 12.14-20.42), ischemic heart disease (OR 3.12, 95\% Cl: 2.16-4.5), female gender (OR 1.56, 95\% Cl: 1.22-2.00), and sudden-onset (OR 1.97, 95\% Cl: 1.54-2.51), were independent significant predictors of cardioembolic stroke. Conclusions: Potential cardioembolic stroke requires a comprehensive evaluation, since early classification and identification through predictors would improve effective management. (REV INVEST CLIN. 2021;73(1):23-30)
\end{abstract}

Key words: Aged. Atrial fibrillation. Intracranial embolism and thrombosis. Risk factors. Stroke/classification. Stroke/etiology.

\section{INTRODUCTION}

Cardioembolic stroke is an important topic on the borderline of geriatric cardiology and vascular neurology.
Cardioembolic infarction accounts for $18-25 \%$ of all ischemic strokes ${ }^{1-4}$ and its incidence is expected to rise because of the age-related incidence of cardiac embolism and an aging population and increasing life expectancy ${ }^{5}$.
*Corresponding author:

Adrià Arboix

E-mail: aarboix@quironsalud.es
Received for publication: 19-05-2020

Approved for publication: 13-07-2020

DOI: $10.24875 / R I C .20000227$

0034-8376 / (c) 2020 Revista de Investigación Clínica. Published by Permanyer. This is an open access article under the CC BY-NC-ND license (http://creativecommons.org/licenses/by-nc-nd/4.0/). 
Basically, there are two etiological types of non-lacunar ischemic stroke: atherothrombotic infarct and cerebral cardioembolism, which cause cardioembolic stroke, the most severe ischemic stroke subtype with high in-hospital mortality rate $(6-27 \%)^{4}$. These two subtypes of ischemic stroke are worth distinguishing since the causes, clinical pictures, outcome, and treatment strategies are different. For instance, oral anticoagulation is indicated for secondary prevention in most patients with cardioembolism without contraindications for anticoagulant therapy whereas antiplatelets, endarterectomy, or angioplasties can be recommended in atherothrombotic stroke.

An early presumptive diagnosis of the stroke subtype can be made following a thorough history, physical examination, and imaging studies such as computed tomography (CT) or magnetic resonance imaging (MRI). However, confirmation of the etiological diagnosis requires more extensive testing.

The aim of this study was identifying the early clinical features related to cardioembolic stroke to help clinicians to improve the adequate and timely management of this neurovascular geriatric ischemic stroke subtype. As a secondary objective, we aimed to compare clinical features and early outcomes of cardioembolic ischemia versus ischemic atherothrombotic stroke to contribute to improve the early clinical differentiation of these two ischemic stroke subtypes.

\section{METHODS}

\section{Setting and study design}

Retrospective clinical study based on prospectively collected data available from an ongoing hospitalbased stroke registry.

Since 1986, the Hospital Universitari Sagrat Cor (an acute-care 350-bed teaching hospital in Barcelona, Catalonia, Spain, serving a population of over 300,000 inhabitants) established a hospital-based Stroke Registry $^{6}$. Data of all patients included in our stroke registry were entered following a standardized protocol with 186 items (demographic features, risk factors, clinical characteristics, laboratory and neuroimaging data, complications, and outcome). The use of the same protocol for all patients ensures completeness of the information in the database. Stroke subtypes are classified according to criteria of the Cerebrovascular Study Group of the Spanish Society of Neurology $^{7}$, which is similar to the National Institute of Neurological Disorders and Stroke classification ${ }^{8}$. The study protocol was approved by the Clinical Research Ethics Committee of the hospital.

\section{Patient selection and study procedures}

The frequency of the stroke subtypes of the registry was as follows: 956 cardioembolic (20.8\%), 945 atherothrombotic (20.5\%), 864 lacunar (18.8\%), 128 unusual cause (2.8\%), 374 essential (8.1\%), 761 transient ischemic attack (TIA) (16.5\%), 473 cranial intracerebral hemorrhage (10.3\%), 52 subarachnoid hemorrhage $(1.1 \%)$, and 43 spontaneous subdural hemorrhage/spontaneous epidural hemorrhage (1\%).

For the purpose of the study, consecutive patients diagnosed with cardioembolic infarction were selected from the database of the Stroke Registry. Criteria for classifying patients as having cardioembolic infarction required the presence of a mediumsized (maximal diameter of the lesion $1.5-3 \mathrm{~cm}$ ) or large $(>3 \mathrm{~cm}$ ) cerebral infarction, cerebral cortex involvement on the brain CT and/or MRI scans, sudden (minutes) or acute (hours) onset, stroke onset during ordinary daily activities, peak of deficit at onset, duration of focal neurological deficit $>24 \mathrm{~h}$, absence of lacunar clinical syndrome, and identification of a commonly accepted cardiac source of embolus in the absence of confirmatory clinical (ipsilateral carotid bruit) or investigative results (Doppler ultrasonography, carotid angiography, or angio-MRI) of lesions (stenosis $\geq 50 \%$ ) in the ipsilateral supraaortic trunks.

Criteria for classifying patients as having atherothrombotic stroke required the presence of a medium or large cerebral infarction, absence of lacunar clinical syndromes documented by CT and/or MRI and evidence of two or more of the following manifestations: (a) presence of carotid, subclavian, vertebral bruit or absent carotid pulses or unequal radial pulses; (b) duplex sonography or angiography, showing arterial stenosis $>50 \%$ or occlusion of the symptomatic artery; and (c) evidence of clinical complications of atherothrombotic disease elsewhere, i.e., angina pectoris, 
previous myocardial infarction, peripheral vascular disease, femoral bruits, and absence of foot pulses.

All patients were admitted to the hospital within 48 hours of the onset of symptoms and did not receive thrombolytic therapy. On admission, demographic characteristics, salient features of clinical, and neurological examination and results of laboratory tests (blood cell count, biochemical profile, serum electrolytes, and urine analysis), chest radiography, 12-lead electrocardiography, and brain CT and/or MRI were recorded, as well as other clinical investigations performed at the discretion of the neurologist in charge. The registry included medical complications - respiratory, cardiac, urinary, renal, and vascular- and mortality during the acute phase of the disease. The degree of clinical disability at discharge from the hospital was evaluated according to the modified Rankin scale?

\section{Statistical analysis}

Continuous data were summarized as mean and standard deviation (SD), and categorical data were summarized as frequency and percentages. The distributions of variables in patients of both groups were compared with the Chi-square $\left(\chi^{2}\right)$ test or the Fisher's exact test for categorical variables and the Student's t-test for quantitative variables. For all analyses, $p<0.05$ was taken to indicate significance.

Covariates with $p<0.20$ in the univariate testing were entered into three multivariable logistic regression models with a stepwise selection method, in which cardioembolic stroke (vs. atherothrombotic infarction) was the dependent variable. Model 1 was based on demographics and cardiovascular risk factors, to which clinical features and vascular topography (model 2) and complications (model 3 ) were added. The odds ratio (OR) and $95 \%$ confidence interval $(\mathrm{Cl})$ were calculated for the final statistically significant variables independently associated with cardioembolic stroke as well as in atherothrombotic stroke. The accuracy of model 3 to identify cardioembolic infarction was assessed using the receiver operating characteristics (ROC) curve. The sensitivity, specificity, and positive and negative predictive values were calculated. Statistical analyses were computed using the package SPSS (Version 20 for Mac; SPSS Inc., Chicago, IL, USA).

\section{RESULTS}

The crude cohort consisted of 4,597 consecutive patients diagnosed with acute stroke. Following etiological investigation, 956 patients were classified as cardioembolic infarction and 945 as atherothrombotic infarction. The results of differences between cardioembolic and atherothrombotic groups by univariate analysis are presented in table 1 . Overall, female gender, 85 years or older, atrial fibrillation (AF), ischemic heart disease, and congestive heart failure were significantly more frequent risk factors in the cardioembolic group, whereas hypertension, diabetes, peripheral vascular disease, heavy smoking, hyperlipidemia, and previous TIA were significantly more frequent in the atherothrombotic group.

Sudden-onset and altered consciousness were significantly more frequent clinical features in the cardioembolic group, and headache, and ataxia were significantly more frequent among atherothrombotic patients. The distribution of lesions according to the vascular topography and medical complications were similar within groups. Early outcomes reported a significant higher percentage of patients with mild neurological deficits at discharge in atherothrombotic infarction compared to cardioembolic patients. The in-hospital mortality rate was also significantly higher in the cardioembolic group than in atherothrombotic patients $(22.8 \%$ vs. $12.7 \%)(p=0.000)$.

The results of multivariate analysis are shown in table 2. The first logistic regression model based on demographics and cardiovascular risk factors reported that AF (OR 15.87), ischemic heart disease (OR 3.11), and female gender (OR 1.58) were independently associated with cardioembolic stroke, whereas hypertension, diabetes, hyperlipidemia, and previous TIA were predictors of atherothrombotic infarcts. In the second regression model, the aforementioned factors (OR 15.75; OR 3.14; OR 1.53, respectively), and sudden-onset (OR 1.98) were predictors of cardioembolic stroke. The third model, in which medical complications were added, reported that AF (OR 15.75), ischemic heart disease (OR 3.12), sudden onset (OR 1.97), cardiac events (OR 1.69), and female gender (OR 1.56) were independent variables related to cardioembolic stroke. Other variables including hypertension, diabetes, hyperlipidemia, visual disturbances, ataxia, infarction in the territory of the anterior 
Table 1. Results of univariate analysis: differences between patients with cardioembolic infarction versus atherothrombotic stroke

\begin{tabular}{|c|c|c|c|}
\hline Variables & $\begin{array}{l}\text { Cardioembolic } \\
(n=956)\end{array}$ & $\begin{array}{l}\text { Atherothrombotic } \\
\quad(n=945)\end{array}$ & $p$ value \\
\hline \multicolumn{4}{|l|}{ Demographics } \\
\hline Female patients & $602(63 \%)$ & $471(49.8 \%)$ & \\
\hline Age, years, mean (SD) & $80(9.14)$ & $77.01(9.75)$ & 0.000 \\
\hline 85 years old or more & $317(33.2 \%)$ & $219(23.2 \%)$ & 0.001 \\
\hline \multicolumn{4}{|l|}{ Risk factors (\%) } \\
\hline Hypertension & $513(53.6)$ & $643(68.0)$ & 0,001 \\
\hline Diabetes mellitus & $177(18.5)$ & $307(32.5)$ & 0,001 \\
\hline Ischemic heart disease & $224(23.4)$ & $66(7.0)$ & 0,001 \\
\hline Atrial fibrillation & $711(74.3)$ & $132(14.0)$ & 0.000 \\
\hline Congestive heart failure & $100(10.4)$ & $48(5.1)$ & 0.000 \\
\hline History of transient ischemic attack (TIA) & $96(10.0)$ & $133(14.1)$ & 0.007 \\
\hline History of cerebrovascular disease & $174(18.2)$ & $190(20.1)$ & 0.286 \\
\hline Previous cerebral hematoma & $11(1.1)$ & $9(1.0)$ & 0.674 \\
\hline Chronic obstructive pulmonary disease & $84(8.8)$ & $97(10.3)$ & 0.269 \\
\hline Chronic renal disease & $57(6.0)$ & $32(3.4)$ & 0.008 \\
\hline Peripheral vascular disease & $91(9.5)$ & $81(8.6)$ & 0.476 \\
\hline Chronic liver disease & $20(2.1)$ & $22(2.3)$ & 0.724 \\
\hline Hyperlipidemia & $130(13.6)$ & $234(24.8)$ & 0.000 \\
\hline Heavy smoking (> 20 cigarettes/day) & $42(4.4)$ & $123(13.0)$ & 0.000 \\
\hline \multicolumn{4}{|l|}{ Clinical features (\%) } \\
\hline Sudden onset & $625(65.3)$ & $401(42.4)$ & 0.000 \\
\hline Headache & $73(7.6)$ & $116(12.3)$ & 0.001 \\
\hline Dizziness & $27(2.8)$ & $51(5.4)$ & 0.005 \\
\hline Early seizures & $17(1.8)$ & $17(1.8)$ & 0.970 \\
\hline Nausea/vomiting & $62(6.5)$ & $84(8.9)$ & 0.048 \\
\hline Altered consciousness & $264(27.6)$ & $186(19.7)$ & 0.000 \\
\hline Motor symptoms & $762(79.6)$ & $718(76.0)$ & 0.056 \\
\hline Sensory symptoms & $346(36.2)$ & $360(38.1)$ & 0.381 \\
\hline Visual disturbances & $202(21.1)$ & $225(23.8)$ & 0.158 \\
\hline Speech disturbances & $589(61.5)$ & $518(54.8)$ & 0.003 \\
\hline Ataxia & $40(4.2)$ & $78(8.3)$ & 0.000 \\
\hline Cranial nerve palsy & $43(4.5)$ & $64(6.8)$ & 0.031 \\
\hline \multicolumn{4}{|l|}{ Vascular topography (\%) } \\
\hline Middle cerebral artery involvement & $620(64.8)$ & $518(54.8)$ & 0.000 \\
\hline Anterior cerebral artery & $45(4.7)$ & $35(3.7)$ & 0.278 \\
\hline Posterior cerebral artery involvement & $77(8.0)$ & $87(9.2)$ & 0.367 \\
\hline Anterior choroidal artery & $4(0.4)$ & $13(1.4)$ & 0.026 \\
\hline Vertebral artery & $19(2.0)$ & $57(6.0)$ & 0.000 \\
\hline Basilar artery & $23(2.4)$ & $91(9.6)$ & 0.000 \\
\hline Internal carotid involvement & $24(2.5)$ & $67(7.1)$ & 0.000 \\
\hline Posteroinferior cerebellar artery & $9(0.9)$ & $15(1.6)$ & 0.206 \\
\hline
\end{tabular}


Table 1. Results of univariate analysis: differences between patients with cardioembolic infarction versus atherothrombotic stroke (continued)

\begin{tabular}{lccc}
\hline Variables & $\begin{array}{c}\text { Cardioembolic } \\
(\mathrm{n}=956)\end{array}$ & $\begin{array}{c}\text { Atherothrombotic } \\
(\mathrm{n}=945)\end{array}$ & $\mathrm{p}$ value \\
\hline Complications (\%) & & & $115(12.2)$ \\
\hline Neurological & $126(13.2)$ & $107(11.3)$ & 0.513 \\
Respiratory & $138(14.4)$ & $14(1.5)$ & 0.044 \\
Renal & $17(1.8)$ & $107(11.3)$ & 0.612 \\
Urinary & $86(9.0)$ & $33(3.5)$ & 0.092 \\
Cardiac events & $86(9.0)$ & $11(1.2)$ & 0.000 \\
Vascular & $27(2.8)$ & $10(1.1)$ & 0.010 \\
Hemorrhagic events & $27(2.8)$ & 0.005 \\
\hline
\end{tabular}

choroidal artery, internal carotid and arterial basilar involvement, urinary complications, and mild neurological deficit at hospital discharge, were independent variables associated with atherothrombotic patients.

According to these models, cases of cardioembolic infarction versus atherothrombotic stroke were correctly classified in $80.5 \%$ of the cases for model 1 , $81.5 \%$ of the cases for model 2 , and $81.8 \%$ of the cases for model 3.

Figure 1 shows the ROC curve of the accuracy of the regression model based on demographics, cardiovascular risk factors, clinical features, vascular topography, and complications. The area under the curve was 0.879 . The sensitivity was $78 \%$, specificity was $85 \%$, positive predictive value was $84 \%$, and negative predictive value was $79 \%$.

\section{DISCUSSION}

The results of the present study confirm that cardioembolic stroke is a severe neurovascular geriatric ischemic stroke subtype that presents a lower frequency of mild functional limitation and higher rates of neurological impairment at hospital discharge, and higher in-hospital mortality, coinciding with other series in the literature ${ }^{10-15}$.

In our study, AF is the most significant cerebrovascular risk factor associated with cardioembolic stroke. This is due to the fact that non-valvular AF is the leading cause of underlying heart disease as stated in different series of cardioembolic infarction ${ }^{14-16}$. Both, permanent and paroxysmal AF increase the risk of cardioembolic stroke ${ }^{17}$. AF leads to inadequate contraction of the atria, resulting in stasis of blood and, consequently, clot formation within the left atrial appendage, with subsequent risk of cerebral embolization. The prevalence of AF is globally increasing caused by improved survival rates of patients with heart disease, and the increase in age of the population. Thus, the number of AF -related strokes may triple in the next decades in high-income countries ${ }^{3}$.

The presence of coronary heart disease and other types of peripheral vascular disease are considered markers of increased risk for subsequent cardiovascular and cerebrovascular events in ischemic stroke $\mathrm{e}^{3,4}$. In our study, ischemic heart disease (found in $23.4 \%$ of patients) was independently associated with cardioembolic stroke in all three logistic regression models $(\mathrm{OR}=3.11$ in the first model; $\mathrm{OR}=3.13$ in the second model and $\mathrm{OR}=3.12$ in the third model). In these cases, isolated ischemic heart disease (with a left ventricular aneurysm, left ventricular ejection fraction $<40 \%$, or with akinesia/dyskinesia of 2 or more segments), or associated with AF was a welldefined structural cardioembolic source. In addition, ischemic heart disease, mainly angor pectoris without associated structural heart disease, may also be a manifestation of generalized atherosclerotic disease and was found in $7 \%$ of patients with atherothrombotic infarctions. However, in these cases, the presence of ischemic heart disease was merely coincidental; it was an epiphenomenon that did not imply a cardioembolic risk. 
Table 2. Results of multivariate analysis: variables independently associated with cardioembolic infarction

\begin{tabular}{lcccc}
\hline Regression models & Coefficient $(\beta)$ & Standard error & $\begin{array}{c}\text { Odds ratio } \\
(95 \% \text { confidence interval) }\end{array}$ & $p$ value \\
\hline First model: demographics and risk factors & & & & \\
\hline Atrial fibrillation & 2.76 & 0.125 & $15.87(12.43-20.27)$ & 0.000 \\
Ischemic heart disease & 1.136 & 0.182 & $3.115(2.179-4.452)$ & 0.000 \\
Female gender & 0.461 & 0.122 & $1.586(1.249-2.013)$ & 0.000 \\
Hyperlipidemia & -0.382 & 0.156 & $0.682(0.502-0.926)$ & 0.014 \\
History of TIA & -0.446 & 0.188 & $0.641(0.443-0.926)$ & 0.018 \\
Diabetes mellitus & -0.637 & 0.139 & $0.529(0.403-0.694)$ & 0.000 \\
Hypertension & -0.678 & 0.125 & $0.508(0.397-0.649)$ & 0.000 \\
\hline
\end{tabular}

Second model: demographics, risk factors, clinical features, and vascular topography

\begin{tabular}{|c|c|c|c|c|}
\hline Atrial fibrillation & 2.757 & 0.131 & $15.749(12.193-20.342)$ & 0.000 \\
\hline Ischemic heart disease & 1.144 & 0.185 & $3.139(2.184-4.512)$ & 0.000 \\
\hline Sudden onset & 0.682 & 0.124 & $1.978(1.552-2.521)$ & 0.000 \\
\hline Female gender & 0.425 & 0.125 & $1.529(1.196-1.956)$ & 0.001 \\
\hline Hyperlipidemia & -0.325 & 0.159 & $0.722(0.529-0.986)$ & 0.040 \\
\hline Diabetes mellitus & -0.58 & 0.142 & $0.560(0.424-0.740)$ & 0.000 \\
\hline Visual disturbances & -0.588 & 0.153 & $0.556(0.412-0.750)$ & 0.000 \\
\hline Hypertension & -0.656 & 0.129 & $0.519(0.403-0.668)$ & 0.000 \\
\hline Ataxia & -0.661 & 0.286 & $0.516(0.295-0.905)$ & 0.021 \\
\hline Basilar artery involvement & -1.098 & 0.299 & $0.334(0.185-0.600)$ & 0.000 \\
\hline Internal carotid involvement & -1.198 & 0.317 & $0.302(0.162-0.562)$ & 0.000 \\
\hline Anterior choroidal artery & -1.886 & 0.74 & $0.152(0.036-0.646)$ & 0.011 \\
\hline
\end{tabular}

Third model: demographics, risk factors, clinical features, vascular topography, and complications

\begin{tabular}{llclc}
\hline Atrial fibrillation & 2.757 & 0.133 & $15.747(12.145-20.417)$ & 0.000 \\
Ischemic heart disease & 1.137 & 0.188 & $3.118(2.157-4.509)$ & 0.000 \\
Sudden onset & 0.676 & 0.125 & $1.966(1.538-2.512)$ & 0.000 \\
Cardiac events & 0.526 & 0.269 & $1.693(0.999-2.869)$ & 0.050 \\
Female gender & 0.445 & 0.127 & $1.56(1.217-2.000)$ & 0.000 \\
Hyperlipidemia & -0.333 & 0.161 & $0.717(0.523-0.983)$ & 0.039 \\
Visual disturbances & -0.57 & 0.156 & $0.566(0.417-0.768)$ & 0.000 \\
Mild neurological deficit at & -0.514 & 0.147 & $0.598(0.448-0.798)$ & 0.000 \\
$\quad$ discharge & -0.57 & 0.156 & $0.566(0.417-0.768)$ & 0.000 \\
Visual disturbances & -0.582 & 0.144 & $0.559(0.422-0.74)$ & 0.000 \\
Diabetes & -0.639 & 0.287 & $0.528(0.301-0.926)$ & 0.026 \\
Ataxia & -0.666 & 0.13 & $0.514(0.398-0.663)$ & 0.000 \\
Hypertension & -1.193 & 0.309 & $0.303(0.166-0.555)$ & 0.000 \\
Basilar artery involvement & -1.24 & 0.318 & $0.289(0.155-0.54)$ & 0.000 \\
Internal carotid involvement & -1.745 & 0.754 & $0.175(0.04-0.766)$ & 0.021 \\
Anterior choroidal artery & & & &
\end{tabular}

Model 1: Hosmer-Lemeshow goodness-of-fit test 0.169 , cardioembolic versus atherothrombotic subjects were correctly classified in $80.5 \%$ of cases. Model 2: Hosmer-Lemeshow goodness-of-fit test 0.239 , cardioembolic versus atherothrombotic subjects were correctly classified in $81.5 \%$ of cases. Model 3: Hosmer-Lemeshow goodness-of-fit test 0.08 , cardioembolic versus atherothrombotic subjects were correctly classified in $81.8 \%$ of cases. 
Figure 1. ROC curve for regression model 3 including demographics, cardiovascular risk factors, clinical features, vascular topography, and complications (AUC: 0.879). AUC: area under the curve; ROC: receiver operating characteristics.

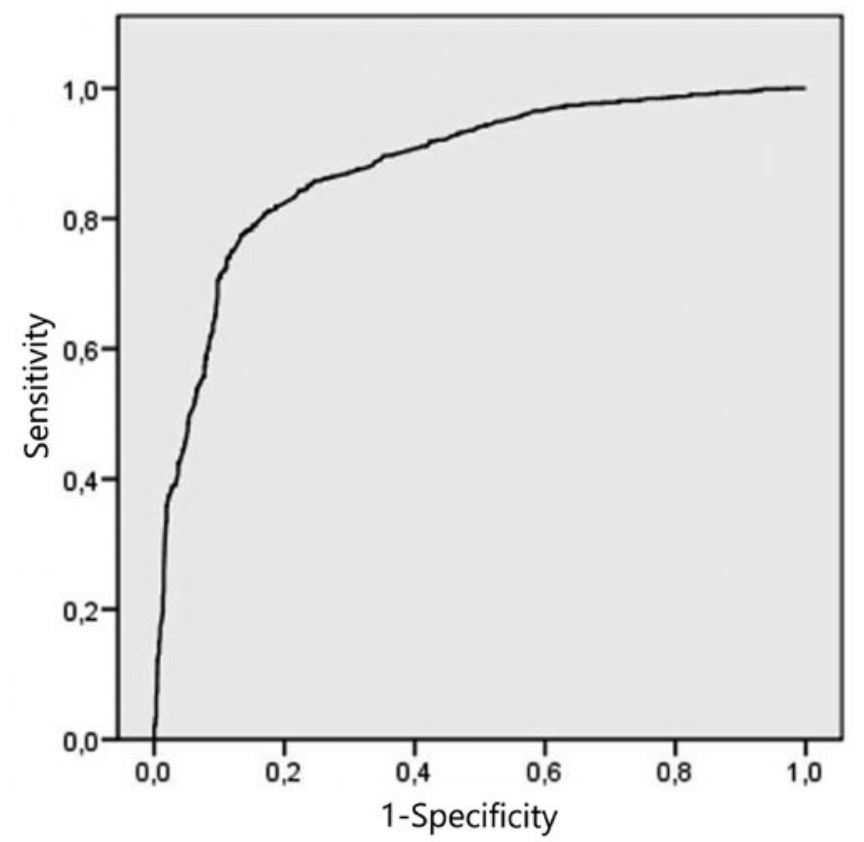

Approximately $2.5 \%$ of patients with acute myocardial infarction experience a stroke within 2-4 weeks of the infarction, and $8 \%$ of men and $11 \%$ of women will have an ischemic stroke within the next 6 years $3,4,17,18$.

Other risk factors for cardiovascular disease such as hypertension, diabetes mellitus, and dyslipidemia are more frequent among atherothrombotic ischemic stroke patients, as reported in the previous studies ${ }^{4,19}$. When all of these risk factors and stroke mechanisms are considered together, they account for $60-80 \%$ of the population-attributable risk of ischemic stroke ${ }^{20}$.

Of interest, female gender is an independent predictor of cardioembolic infarction in all three regression models, as reported in literature, possibly due to the longer life expectancy of women, and this higher biological age may lead to an increased likelihood of developing $\mathrm{AF}$, ischemic heart disease, and other related heart diseases ${ }^{21-23}$.

Sudden onset to maximal deficit and non-lacunar clinical syndrome are also significant predictors of cardioembolic infarction $4,24,25$. This is in line with the study of Timsit et al., who reported that sudden onset of neurological deficit was more frequent in cardioembolic stroke (79\%) than in lacunar (38\%) or atherothrombotic infarction $(46 \%)^{26}$. The embolus blocks suddenly so that the onset of symptoms is abrupt and neurological deficits are maximal at this moment. Conversely, visual disturbances and ataxia are more frequent among atherothrombotic stroke patients.

It is noteworthy that vascular involvement of the basilar trunk, the internal carotid artery and the anterior choroidal artery are less frequent in cardioembolic infarction compared to thrombotic infarction. This might be due to the fact that cerebral embolism usually occludes more distal cerebral arteries, such as the middle cerebral artery or the posterior cerebral artery ${ }^{4}$. In contrast, hemodynamically significant stenosis at the supra-aortic trunk level or at the vertebrobasilar territory level is common in atherothrombotic infarction.

It should be noted that our results confirm that cardioembolic stroke is the most severe subtype of ischemic stroke, with a high mortality rate, and a higher rate of complications, particularly cardiac events ${ }^{27,28}$ compared to lacunar infarctions and the other subtypes of cerebral ischemia ${ }^{24}$. Appropriate treatment measures are essential for secondary stroke prevention, as many cardiac conditions pose a high stroke recurrence risk. In cardioembolic stroke, oral anticoagulation should be started according to the severity of the infarction, and its cerebral extension ${ }^{1-3}$. 
The comparative study of the evolution of demographic characteristics and clinical and outcome data in patients with cardioembolic stroke overtime was not the aim of this study. However, this evolution in our stroke registry was already analyzed in a previous study in which, over a 19-year study period, we observed significant changes in patients with a first-ever cardioembolic stroke that included an increase in the age of patients (the percentage of very old patients increased from $16 \%$ to $38.2 \%$ ), in frequency of hypertension (from $40.5 \%$ to $60.8 \%$ ), and use of echocardiography (from $39.7 \%$ to $73.9 \%$ ), while the frequency of heavy smoking $>20$ cigarettes/day (from $9.2 \%$ to $2 \%$ ) and length of hospital stay decreased ${ }^{29}$. It should be noted that the lack of improvement in the early prognosis of patients with cardioembolic stroke during this study period (both mortality and neurological impairment) can be explained by the increased prevalence of major cardiovascular risk factors due to progressive ageing of the population ${ }^{29}$.

Limitations of the present study include the retrospective analysis of data based on a single-center stroke registry, and hospital referral selection bias cannot be excluded. Laboratory, neuroimaging, and neuropsychological variables were not included in multivariate analysis, which would have provided a more robust regression model ${ }^{30}$. Strengths of the study are the large number of patients analyzed the systematic evaluation of predictors of cardioembolic infarction based on risk factors, clinical features, vascular topography, and outcome from a previously validated stroke registry.

We conclude that cardioembolic stroke is a geriatric neurovascular entity, and its early identification is important in triaging medical resources, choosing among treatment modalities, and predicting clinical outcomes.

\section{REFERENCES}

1. Cadilhac DA, Kim J, Lannin NA, Kapral MK, Schwamm LH, Dennis MS, et al. National stroke registries for monitoring and improving the quality of hospital care: a systematic review. Int J Stroke. 2016;11:28-40.

2. Ferro JM. Brain embolism. Answers to practical questions. J Neurol. 2003;250:139-47.

3. Kamel H, Healey JS. Cardioembolic stroke. Circ Res. 2017; 120:514-26.

4. Arboix A, Alió J. Acute cardioembolic stroke: an update. Expert Rev Cardiovasc Ther. 2011;9:367-79.

5. Carbajo-García AM, Cortés J, Arboix A, Massons J, Díez L, Vergés $\mathrm{E}$, et al. Predictive clinical features of cardioembolic infarction in patients aged 85 years and older. J Geriatr Cardiol. 2019;16:793-9.

6. Arboix A, Massons J, Oliveres M, García L, Titus F. An analysis of 1000 consecutive patients with acute cerebrovascular dis- ease. The registry of cerebrovascular disease of La Alianzahospital central of Barcelona. Med Clin (Barc). 1993;101:281-5.

7. Arboix A, Alvarez-Sabin J, Soler L. Stroke. Classification and diagnostic criteria. Ad hoc editorial committee of the task force on cerebrovascular diseases of SEN. Neurologia. 1998;13:s3-10.

8. Special report from the national institute of neurological disorders and stroke: classification of cerebrovascular diseases III. Stroke. 1990;21:637-76.

9. Bamford JM, Sandercock PA, Warlow CP, Slattery J. Interobserver agreement for the assessment of handicap in stroke patients. Stroke. 1989;20:828.

10. Arboix A, Bechich S, Oliveres M, García-Eroles L, Massons J, Targa C. Ischemic stroke of unusual cause: clinical features, etiology and outcome. Eur J Neurol. 2001;8:133-9.

11. Amin H, Nowak RJ, Schindler JL. Cardioembolic stroke: practical considerations for patient risk management and secondary prevention. Postgrad Med. 2014;126:55-65.

12. Arboix A, García-Eroles L, Massons J, Oliveres M. Predictive clinical factors of in-hospital mortality in 231 consecutive patients with cardioembolic cerebral infarction. Cerebrovasc Dis. 1998;8:8-13.

13. Arboix A, Vericat MC, Pujades R, Massons J, García-Eroles L, Oliveres M. Cardioembolic infarction in the Sagrat Cor-Alianza hospital of Barcelona stroke registry. Acta Neurol Scand. 1997; 96:407-12.

14. Soler EP, Felip PF, Ruiz VC, Orgaz AC, Cartagena PS, Batiste DM. The Mataró stroke registry: a 10-year registry in a community hospital. Neurologia. 2015;30:283-9.

15. Russo T, Felzani G, Marini C. Stroke in the very old: a systematic review of studies on incidence, outcome, and resources. J Aging Res. 2011;2011:108785.

16. Arboix A, Oliveres M, Massons J, Pujades R, Garcia-Eroles L. Early differentiation of cardioembolic from atherothrombotic cerebral infarction: a multivariate analysis. Eur J Neurol. 1999;6:677-83.

17. Moschini J, Lindenbaum S, Rodríguez G. Accidente cerebrovascular isquémico cardioembólico: diagnóstico de trombo en la orejuela auricular izquierda con tomografía computada cardíaca. Neurol Arg. 2013;5:276-9

18. Díaz-Guzmán J, Egido JA, Gabriel-Sánchez R, Barberá-Comes G, Fuentes-Gimeno B, Fernández-Pérez $C$, et al. Stroke and transient ischemic attack incidence rate in Spain: the IBERICTUS study. Cerebrovasc Dis. 2012;34:272-81.

19. Arboix A, Roig H, Rossich R, Martínez EM, García-Eroles L. Differences between hypertensive and non-hypertensive ischemic stroke. Eur J Neurol. 2004;11:687-92.

20. Hankey GJ. Potential new risk factors for ischemic stroke: what is their potential? Stroke. 2006;37:2181-8.

21. Luy M, Gast K. Do women live longer or do men die earlier? Reflections on the causes of sex differences in life expectancy. Gerontology. 2014;60:143-53.

22. Kobayashi LC, Beeken RJ, Meisel SF. Biopsychosocial predictors of perceived life expectancy in a national sample of older men and women. PLoS One. 2017;12:e0189245.

23. Arboix A, Cartanyà A, Lowak M, García-Eroles L, Parra O, Oliveres $M$, et al. Gender differences and woman-specific trends in acute stroke: results from a hospital-based registry (19862009). Clin Neurol Neurosurg. 2014;127:19-24.

24. Arboix A, Blanco-Rojas L, Martí-Vilalta JL. Advancements in understanding the mechanisms of symptomatic lacunar ischemic stroke: translation of knowledge to prevention strategies. Expert Rev Neurother. 2014;14:261-76.

25. Carrera E, Maeder-Ingvar M, Rossetti AO, Devuyst G, Bogousslavsky J, Lausanne Stroke Registry. Trends in risk factors, patterns and causes in hospitalized strokes over 25 years: the Lausanne stroke registry. Cerebrovasc Dis. 2007;24:97-103.

26. Timsit SG, Sacco RL, Mohr JP, Foulkes MA, Tatemichi TK, Wolf $\mathrm{PA}$, et al. Early clinical differentiation of cerebral infarction from severe atherosclerotic stenosis and cardioembolism. Stroke. 1992;23:486-91

27. Kumar S, Selim M, Caplan L. Medical complications after stroke. Lancet Neurol. 2010:9:105-18.

28. Arboix A, García-Eroles L, Oliveres M, Comes E, Sánchez MJ, Massons J. Malignant middle cerebral artery infarction: a clinical study of 32 patients. Rev Invest Clin. 2015;67:64-70.

29. Arboix A, Massons J, Garcia-Eroles L, Targa C, Parra O, Oliveres $M$. Trends in clinical features and early outcome in patients with acute cardioembolic stroke subtypes over a 19-year period. Neurol India. 2012;60:288-93.

30. Blanco-Rojas L, Arboix A, Canovas D, Grau-Olivares M, Morera JC, Parra O. Cognitive profile in patients with a first-ever lacunar infarct with and without silent lacunes: a comparative study. BMC Neurol. 2013;13:203. 